\title{
A steady-state Bi-substrate technique for measurement of the thermal conductivity of ceramic coatings
}

\author{
J.C. Tan, S.A. Tsipas, I.O. Golosnoy, J.A. Curran, S. Paul, T.W. Clyne* \\ Department of Materials Science and Metallurgy, Cambridge University, Pembroke Street, Cambridge CB2 3QZ, UK
}

Received 21 May 2005; accepted in revised form 6 February 2006

Available online 20 March 2006

\begin{abstract}
This paper presents a steady-state, bi-substrate technique for measurement of the through-thickness thermal conductivity of ceramic coatings, with a range of specimen thickness and porosity content. The technique is based on establishing unidirectional steady-state heat flow through the sample, sandwiched between a pair of (metallic) substrates with known thermal properties. Comparison between the heat fluxes passing through the two substrates allows a check to be made about the accuracy of the assumption of unidirectional heat flow. The interfacial conductances must be known and these can be estimated by testing samples of different thickness. Measured conductivities are likely to be more accurate if the interfacial conductance is relatively high. This is assisted by the introduction of a thin interfacial layer of a viscous, thermally conductive compound, or thermal pads of some sort, and by maintaining a suitable pressure across the setup. However, if such compounds (pastes) are used, then care must be taken to ensure that it does not enter the specimen via surface-connected pores, since this could significantly affect the measured conductivity. The reliability of the technique has been confirmed by testing fused silica samples of known thermal conductivity. It has also been applied to sprayed zirconia and plasma electrolytic oxide (PEO) alumina coatings. The values obtained were $1.05 \pm 0.10 \mathrm{~W} \mathrm{~m}^{-1} \mathrm{~K}^{-1}$ and $1.63 \pm$ $0.35 \mathrm{~W} \mathrm{~m}^{-1} \mathrm{~K}^{-1}$, respectively.
\end{abstract}

(C) 2006 Published by Elsevier B.V.

Keywords: Thermal conductivity; Interfacial thermal conductance; Ceramic coating; Plasma sprayed zirconia; Thermal barrier coating; Plasma electrolytic oxide coating

\section{Introduction}

Several experimental techniques have been developed for measurement of thermal conductivity, applicable over a wide range of temperature [1] and for various specimen shapes and dimensions. However, values obtained using different techniques often show significant variations. This is frequently attributable to inaccuracies in the assumed boundary conditions [2]. Most of the techniques currently employed for coatings are either steady-state [3-5] or transient (e.g. laser flash $[6,7])$ methods. Laser flash is often considered to be the most convenient and accurate method. It is quick, applicable over a wide range of temperature, does not require large samples and can be employed under vacuum and in controlled atmospheres. However, there are several question marks associated with the application of this method to materials

\footnotetext{
* Corresponding author.

E-mail address: twc10@cam.ac.uk (T.W. Clyne).
}

such as thermal barrier coatings (TBCs) [6,7]. One problem lies in determining suitable laser pulse power and duration values [7]. Another concerns the semi-transparency of many ceramics (zirconia, alumina, etc.) at wavelengths typical of lasers and infrared detectors. In order to improve radiation absorbtion, thin surface layers, for example of carbon, gold or platinum, are commonly employed. However, high temperatures can cause evaporation of these layers and possible reaction with the sample is also a potential cause for concern [8]. Also, since the laser flash method actually measures the thermal diffusivity of the sample, calculation of conductivity requires prior knowledge of the volume specific heat at different temperatures. Since TBCs often exhibit large variations in porosity and crystallite size $[9,10]$, it can be difficult to evaluate the volume specific heat and use of handbook data may be unreliable.

With steady-state methods, on the other hand, just the temperature drop across the sample and the heat flux are required to obtain the thermal conductivity. This is a well- 
established approach for bulk material, although there is always concern about lateral heat losses and maintenance of unidirectional heat flow conditions. However, there are many situations in which the material of interest is not available in bulk forman obvious example being surface coatings. Moreover, the thermal conductivity of such materials is sometimes of prime importance, particularly for thermal barrier coatings. Steady state measurement can be carried out with thin specimen of this type by locating it between two metallic blocks. Relatively little has been published about such a procedure, although the method has been used previously $[4,11]$. This paper presents an analysis of the method and a brief study of the issues affecting accuracy and reliability.

\section{A steady state Bi-substrate thermal conductivity measurement technique}

\subsection{Experimental setup}

Fig. 1(b) shows a schematic of the setup. The sample, in this case with a cross section of $35 \times 30 \mathrm{~mm}$, is sandwiched between two metallic substrates. Various metals can be used, but those with a relatively low thermal conductivity offer the advantage of promoting larger differences between thermocouple readings and hence a more accurate measurement of the heat flux. Nimonic 80A alloy (Special Metals Limited, Hereford, UK) was used in the present work. These substrates act as flux

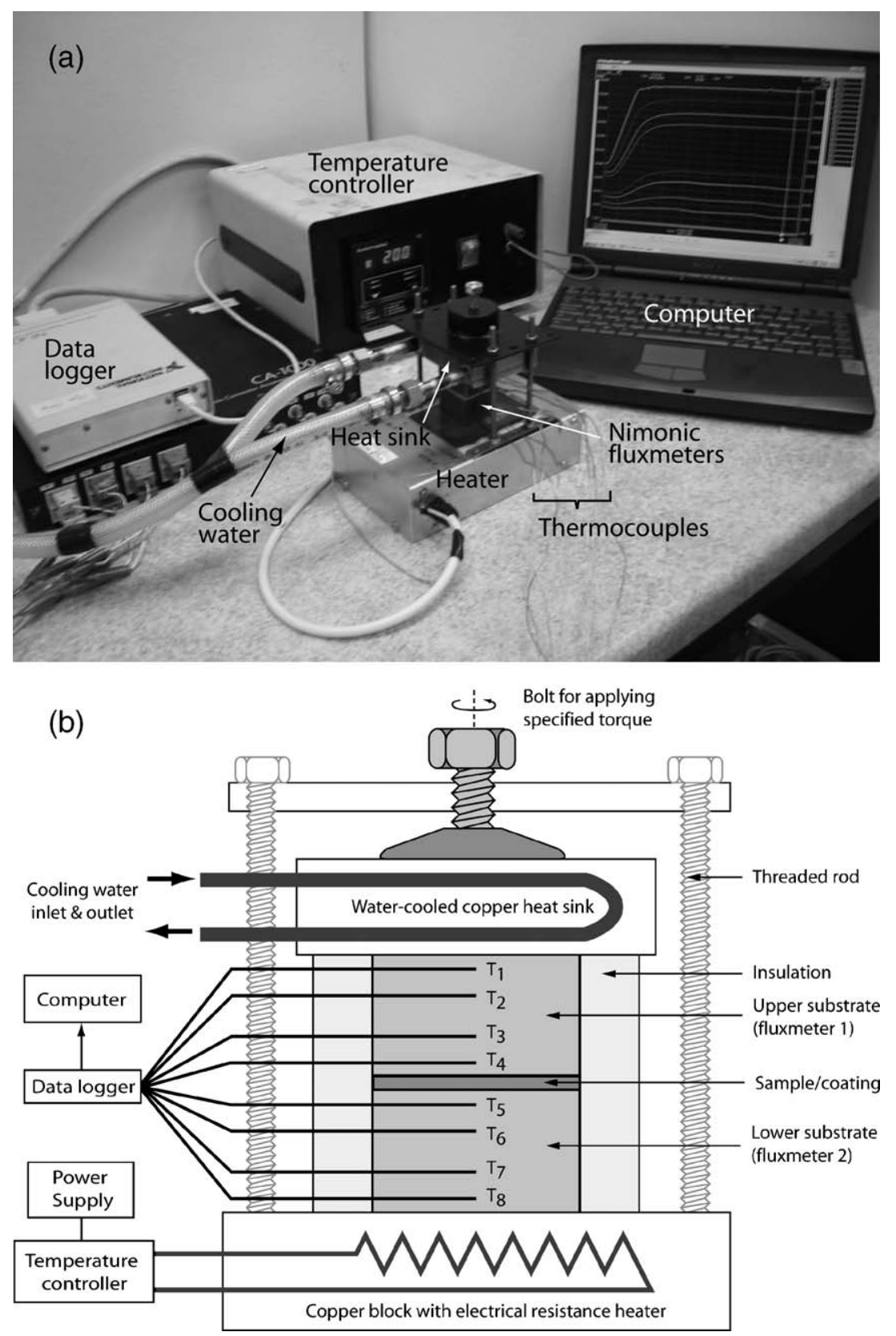

Fig. 1. (a) Photograph of the setup, with the insulation removed. (b) Schematic, showing the eight thermocouples used for monitoring the temperature in the two substrates (flux meters). 
meters, each being instrumented with four K-type thermocouples $\left(T_{1}\right.$ to $\left.T_{8}\right)$, inserted into holes at known distances, drilled to the centerline of each block. A minimum of two temperature readings are required from each substrate to determine the heat flux, but additional values allow its average value to be obtained more accurately and a check to be made on the validity of the assumption of uni-directional heat flow. The (temperature-dependent) thermal conductivity of Nimonic $80 \mathrm{~A}$ is well known over a wide temperature range (Table 1) and this information is used in measuring the heat flux across each substrate. The blocks are insulated laterally with a suitable ceramic fibre blanket material.

Temperature gradients are generated by heating the lower substrate with an electric resistance heater, while heat is continuously removed from the upper substrate via a watercooled copper heat sink. In order to maintain constant rates of heat injection and removal, the heater power and cooling water flow rate are fixed and the system is left for a suitable time to equilibrate, with the thermocouple outputs being recorded. The validity of the assumption of uni-directional heat flow is checked by comparing the average heat fluxes through the two substrates. In general, it is possible to ensure that these fluxes are within $5 \%$ of each other and this is considered to represent a sufficiently accurate approximation to unidirectionality.

To minimize interfacial air gaps and maximise the interfacial thermal conductance $(h)$, two types of substrate-to-sample interfacial layers were tested: (i) a silicone-based thermal compound (HTSP Electrolube), with a conductivity of $3.0 \mathrm{~W} \mathrm{~m}^{-1} \mathrm{~K}^{-1}$ and (ii) Sil-Pad ${ }^{\circledR} 2000$ (Bergquist), a high performance conductive pad, with a thickness of $0.38 \mathrm{~mm}$ and a conductivity of $3.5 \mathrm{~W} \mathrm{~m}^{-1} \mathrm{~K}^{-1}$. To promote reproducibility, a specified torque ( $3 \mathrm{~N} \mathrm{~m}$ in these experiments) was applied via the bolt. This torque generated an axial force of $750 \mathrm{~N}$, corresponding to an applied pressure of $\sim 0.7 \mathrm{MPa}$.

During the experiment, the temperature readings $\left(T_{1}\right.$ to $\left.T_{8}\right)$ were continuously logged. A steady state was considered to have been established when the temperature fluctuations were within $\pm 0.5^{\circ} \mathrm{C}$, for more than $30 \mathrm{~min}$. These steady state temperature data were used to calculate the thermal conductivity of the sample.

Table 1

Temperature-dependent thermal conductivities of materials used as substrates

\begin{tabular}{llll}
\hline Temperature, & \multicolumn{2}{l}{ Thermal conductivity, $K\left(\mathrm{~W} \mathrm{~m}^{-1} \mathrm{~K}^{-1}\right)$} & \\
\cline { 2 - 4 }$T\left({ }^{\circ} \mathrm{C}\right)$ & Nimonic 80A [16] & Fused silica [12] & Al (6082-T6) [17] \\
\hline 0 & & & \\
20 & 11.2 & 1.38 & 172 \\
100 & 12.8 & 1.46 & \\
200 & 14.4 & 1.55 & \\
300 & 16.1 & 1.67 & \\
400 & 17.8 & 1.84 & \\
500 & 19.4 & & \\
600 & 20.8 & & \\
700 & 22.3 & & \\
800 & 24.5 & & \\
900 & 26.5 & & \\
950 & & & \\
1000 & 28.4 & & \\
\hline
\end{tabular}

\subsection{Data analysis}

By assuming one-dimensional heat flow across the setup, and by taking into account changes in thermal conductivity of the blocks with temperature, the mean heat flux, $Q$, can be found using Eqs. (1)-(3):

$Q_{\text {upper }}=\frac{1}{6} \sum_{\substack{j=2 \\ j>k}}^{4} \sum_{k=1}^{3}\left(\frac{T_{j}-T_{k}}{x_{j}-x_{k}}\right) K_{\text {sub }}\left(T_{\text {ave }}^{j k}\right)$,
$T_{\text {ave }}^{j k}=\frac{T_{j}+T_{k}}{2}$

$Q_{\text {lower }}=\frac{1}{6} \sum_{\substack{j=6 \\ j>k}}^{8} \sum_{\substack{k=5 \\ T_{j}-x_{k}}}^{7}\left(\frac{T_{j}-T_{k}}{x_{j}} K_{\text {sub }}\left(T_{\text {ave }}^{j k}\right)\right.$,

$T_{\mathrm{ave}}^{j k}=\frac{T_{j}+T_{k}}{2}$

where $j$ and $k$ designate locations of thermocouples (Fig. 1), while $K_{\text {sub }}\left(T_{\text {ave }}^{j k}\right)$ is the temperature-dependent thermal conductivity of the (Nimonic) substrate, at the average temperature. The mean heat flux can be written as:

$Q=\frac{1}{2}\left(Q_{\text {upper }}+Q_{\text {lower }}\right)$

The conditions are presumed to approximate to onedimensional heat flow when:

$\frac{\left|Q_{\text {upper }}-Q_{\text {lower }}\right|}{Q} \leq 10 \%$

For a typical experiment, this was satisfied quite comfortably with the set-up employed. In general, conditions approach the ideal of unidirectional heat flow more closely as the aspect ratio (lateral/axial dimensions) of the set-up becomes large and as the lateral heat losses are reduced (by use of suitable insulation). The axial extent of the substrates should be reasonably large, so as to accommodate a suitable number of thermocouples (positioned with good precision in terms of axial location), so it follows that specimens with relatively large lateral dimensions are preferred. However, in practice it is relatively easy to ensure approximately unidirectional heat flow, provided the specimens (coatings) have lateral dimensions of the order of at least about $10 \mathrm{~mm}$.

Fig. 2 shows a schematic of the temperature profile established across the substrates and sample (coating) under steady-state conditions. Assuming no lateral heat losses, the same flux flows through coating and interfaces, so that:

$Q=K_{\text {eff }} \frac{\Delta T}{\Delta x}$

$Q=h \Delta T_{\mathrm{i}}$

$Q=K_{\text {true }} \frac{\Delta T_{\mathrm{c}}}{\Delta x}$

where $K_{\text {eff }}$ is the effective thermal conductivity of the coating, $\Delta T$ is the total temperature drop, $\Delta x$ is the coating thickness, $h$ is the interfacial thermal conductance (assumed the same for both 


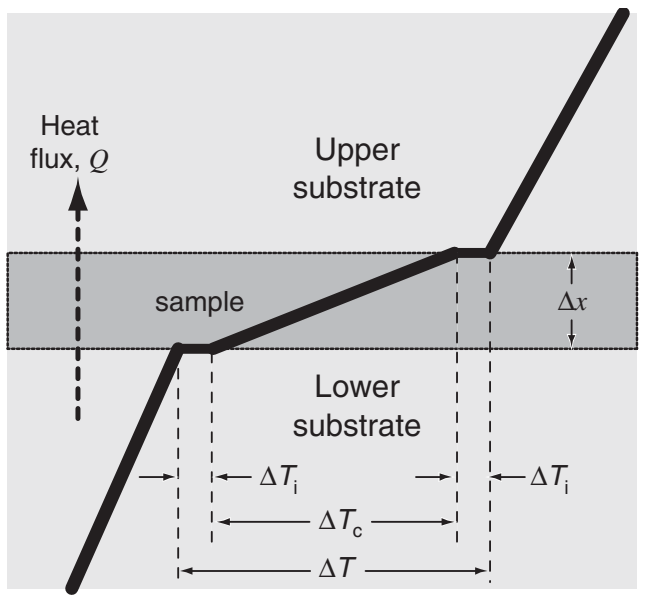

Fig. 2. Schematic depicting the temperature drops across substrates and sample (coating), for a case where there are 2 interfaces $(m=2)$.

interfaces) and $K_{\text {true }}$ is the actual coating thermal conductivity. The subscripts $i$ and $\mathrm{c}$ denote interface and coating, respectively.

The total temperature drop, $\Delta T$ is composed of drops across the coating and across the interfaces

$\Delta T=\Delta T_{\mathrm{c}}+m \Delta T_{\mathrm{i}}$

where $m$ is the number of (identical) interfaces present in the system. By substituting Eqs. (6) and (7) into Eq. (8), and rearranging,

$\frac{\Delta T}{Q}=\frac{\Delta x}{K_{\text {true }}}+\frac{m}{h}$

Plotting $\Delta T / Q$ versus $\Delta x$, the slope and intercept are given by $1 / K_{\text {true }}$ and $m / h$, respectively. Both $K_{\text {true }}$ and $h$ can thus be determined from such a plot (i.e. from data for specimens of different thickness).

\subsection{Validation of technique}

Before the technique can be used with confidence, it must be verified using a standard material of known thermal conductivity. Fused silica (quartz glass) samples supplied by Heraeus Quarzglas (Germany) were chosen for this purpose. In the manufacturer's material datasheet (Table 1), its thermal conductivity is reported as $1.46 \mathrm{~W} \mathrm{~m}^{-1} \mathrm{~K}^{-1}$ at $100^{\circ} \mathrm{C}$ [12]. Samples with three different thicknesses, i.e. 0.567, 0.989 and $2.897 \mathrm{~mm}$, were used. Although the as-received materials had flat and smooth surfaces, additional polishing was performed, to ensure that all samples had a consistent surface finish $(\sim 1 \mu \mathrm{m})$. The silicone-based conductive compound was smeared over the surfaces and pressure applied via the bolt. Fig. 3 shows the steady state temperature versus distance plot for a silica sample with a thickness of $2.897 \mathrm{~mm}$. The mean temperature of the sample was about $100^{\circ} \mathrm{C}$ and the total temperature drop $(\Delta T)$ across the sample was about $48^{\circ} \mathrm{C}$. Using Eqs. (1)-(3), the mean heat flux was estimated as $26.4 \mathrm{~kW} \mathrm{~m}^{-2}$. From Eq. (5), the effective thermal conductivity $\left(K_{\text {eff }}\right)$ was found to be $1.25 \mathrm{~W} \mathrm{~m}^{-1} \mathrm{~K}^{-1}$.

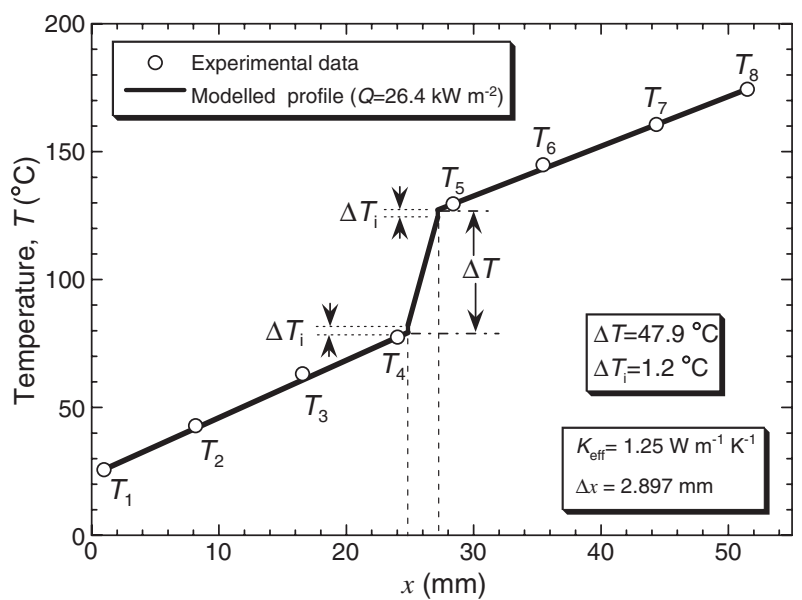

Fig. 3. Temperature versus distance plot, showing the temperature profile across a fused silica sample. The line was obtained using the average heat flux, $Q$, and the temperature-dependent thermal conductivity of Nimonic (Table 1).

Similar profiles were observed for the thinner silica samples, but with smaller temperature drops across the samples. Fig. 4 shows experimental data for all of the fused silica samples, along with the best linear fit, as given by Eq. (9). It can be seen that the data exhibit relatively little scatter. The values of actual thermal conductivity $\left(K_{\text {true }}\right)$ and interfacial thermal conductance (h) were found to be $1.54 \mathrm{~W} \mathrm{~m}^{-1} \mathrm{~K}^{-1}$ and $12.9 \mathrm{~kW} \mathrm{~m}^{-2} \mathrm{~K}^{-1}$, respectively. The measured conductivity agrees quite well with the value $\left(1.46 \mathrm{~W} \mathrm{~m}^{-1} \mathrm{~K}^{-1}\right)$ quoted by the manufacturer [12]. It may also be noted that the interfacial contact conductance measured here is of a similar order of magnitude to values reported previously (for a similar surface finish and contact pressure) [13].

\section{Thermal conductivity of as-sprayed YSZ coatings}

Samples were made of as-sprayed yttria-stabilised zirconia (YSZ, $\mathrm{ZrO}_{2}-8$ wt. $\% \mathrm{Y}_{2} \mathrm{O}_{3}$ ) thermal barrier coatings (TBC). The powders, designated 204-NS, were supplied by Sulzer Metco

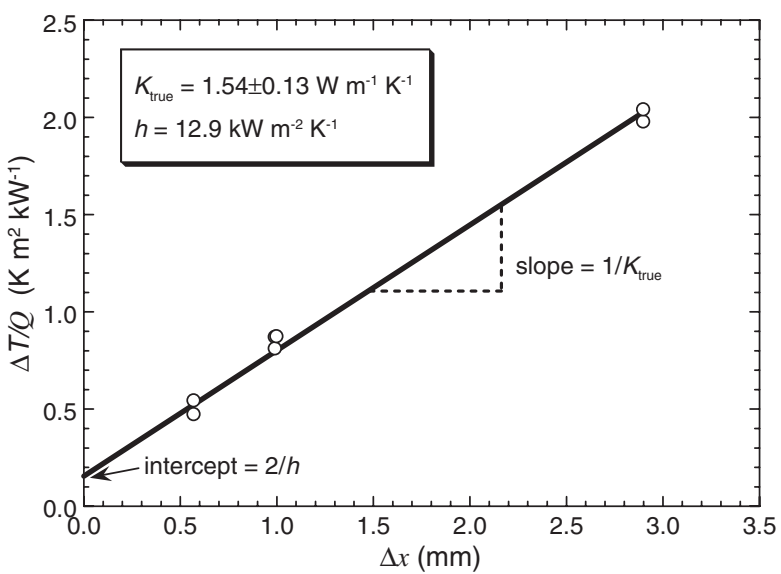

Fig. 4. A plot of $\Delta T / \mathrm{Q}$ versus $\Delta x$ for fused silica samples (three different thicknesses). The points are experimental data, while the line is the best linear fit, using Eq. (9). The values of $K_{\text {true }}$ and $h$ were obtained from the slope and intercept, respectively. 
(Westbury, NY). Specimens were produced by air plasma spraying (APS) onto mild steel substrates of $1.5 \mathrm{~mm}$ thickness. Details of the spraying conditions can be found elsewhere [14]. Samples were prepared by debonding the top coats from their substrates and polishing to $\sim 1 \mu \mathrm{m}$ surface finish. The debonded coatings had thicknesses ranging from 0.5 to $2.0 \mathrm{~mm}$. The porosity levels in material of this type have been measured previously and are typically around $10-15 \%$.

Fig. 5 shows experimental data, and corresponding best linear fits (from Eq. (9), with $m=2$ ), for samples tested using (a) the silicone-based thermal compound and (b) the conductive pads. It is clear that the TBC specimens exhibited greater scatter than the fused silica samples (Fig. 4). The detached YSZ coatings were slightly curved, as a result of residual stresses, making preparation of samples with flat surfaces difficult. Also, the inherent variability in the microstructure of such material is much greater than that of fused silica.

Using the silicone compound, $K_{\text {true }}$ and $h$ were found to be $1.38 \pm 0.27 \mathrm{~W} \mathrm{~m}^{-1} \mathrm{~K}^{-1}$ and $16.7 \mathrm{~kW} \mathrm{~m}^{-2} \mathrm{~K}^{-1}$, respectively (Fig. 5(a)). Using the conductive pads, on the other hand, measured $K_{\text {true }}$ and $h$ values were found to be lower, i.e. 1.05 \pm $0.10 \mathrm{~W} \mathrm{~m}^{-1} \mathrm{~K}^{-1}$ and $4.32 \mathrm{~kW} \mathrm{~m}^{-2} \mathrm{~K}^{-1}$, respectively (Fig. 5 (b)). The thermal compound is apparently more effective than the conductive pads in raising the interfacial thermal conductance $(h)$, presumably by effectively filling gaps between the matching surfaces. However, use of the two types of interfacial layer gives different measured conductivities, with the value
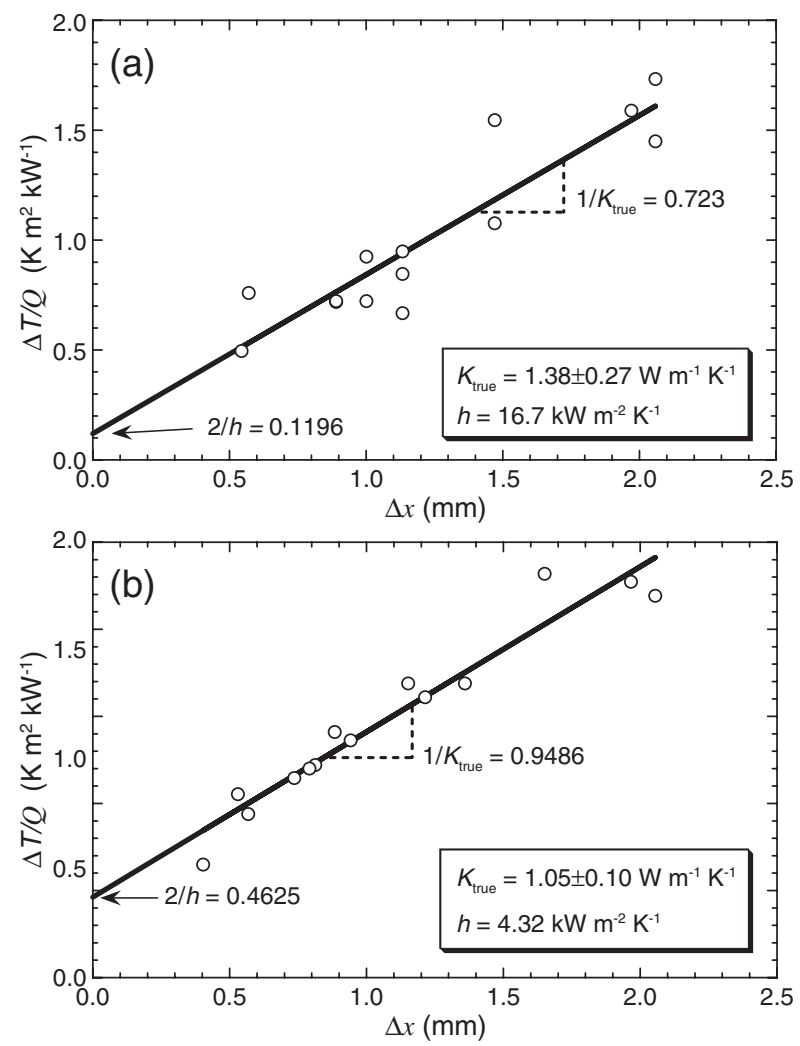

Fig. 5. Plots of $\Delta T / \mathrm{Q}$ versus $\Delta x$ for as-sprayed PSZ coatings, with (a) conductive paste and (b) conductive pads at the interfaces. The values of $K_{\text {true }}$ and $h$ were obtained from the slope and intercept, respectively. obtained using the compound being about $30 \%$ higher. This is attributed to the compound entering the pores and hence raising the apparent conductivity. This was confirmed by microstructural examination. Conductive pads, rather than soft compounds, are therefore recommended for materials, such as sprayed YSZ, with extensive surface-connected porosity. The figure of $1.05 \mathrm{~W} \mathrm{~m}^{-1} \mathrm{~K}^{-1}$ is close to the values obtained for these coatings using laser flash and hot disk techniques.

\section{Thermal conductivity of plasma electrolytic oxide coatings on aluminium}

The technique was also applied to plasma electrolytic oxide (PEO) coatings. These were grown on aluminium, using high voltage pulses to generate multiple through-thickness plasma discharges. Coatings of up to $500 \mu \mathrm{m}$ in thickness are possible, although it is more typical for them to be up to around $100 \mu \mathrm{m}$ thick [15]. It is difficult to detach such coatings without causing physical damage to them, since they are highly adherent (having been formed by partial consumption of the substrate). Furthermore, X-ray diffraction and nano-indentation studies have revealed layers of variable phase composition and physical properties. This complicates the evaluation of properties such as density and specific heat, making transient methods of thermal conductivity measurement particularly problematic. In addition, such thin and partly amorphous coatings have significant transparency to most laser wavelengths, making the use of opaque surface layers essential for any laser-flash measurements.

Clearly, the presence of a strongly adhered substrate, in the same configuration as would be used in practice, lends itself well to a technique that employs such substrate blocks as flux meters. It is also expected that such coatings would only be used at temperatures that the (aluminium) substrate can withstand, so there is no interest in making measurements at very high temperatures. The present technique therefore appears to be well-suited to determining the thermal conductivity of these coatings.

PEO coatings were grown on Al-6082 substrates and their free surfaces were polished, reducing the surface roughness to $\sim 1 \mu \mathrm{m}$. The roughness of the coating/substrate interface was $\sim 4 \mu \mathrm{m}$. Coating thickness was measured with a calibrated eddy current thickness gauge (Oxford Instruments CMI 100). Pairs of coated aluminium blocks (cylinders with $30 \mathrm{~mm}$ diameter and $20 \mathrm{~mm}$ height) were placed back to back, in order to double the coating thickness and hence improve the accuracy. The interfaces between the coatings and their host substrates were considered to have infinite conductance, so that there was effectively only one interface in this case $(m=1)$. Silicone-based thermal compound was used to fill the gap between the coatings. In this case, while these coatings do contain pores, most of them are very fine and little or no penetration of compound into them occurred. Conductive pads, on the other hand, were found to be unsuitable for such thin coatings $(<100 \mu \mathrm{m})$, since they introduced a relatively large interfacial thermal resistance.

Fig. 6 shows data for specimens with a range of thickness $(40-100 \mu \mathrm{m})$, including the best linear fit, obtained 


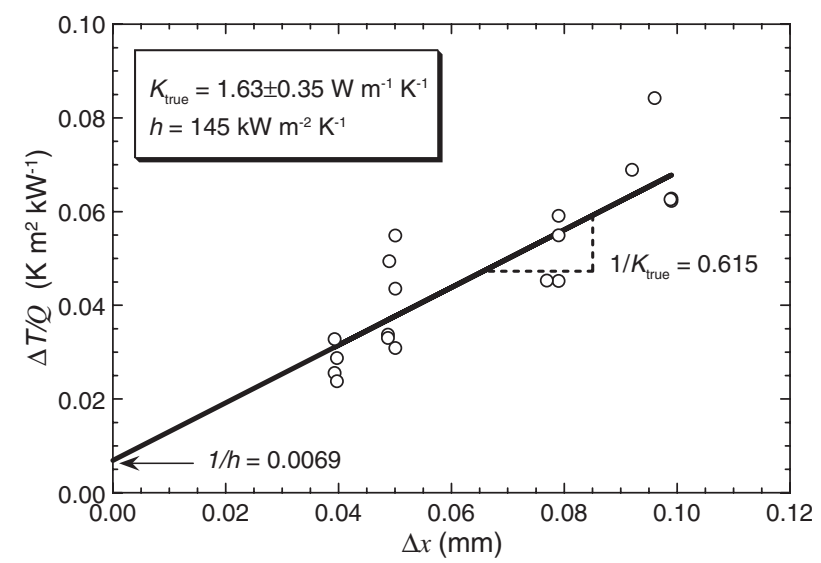

Fig. 6. Plots of $\Delta T / \mathrm{Q}$ versus $\Delta x$ for plasma electrolytic oxide (PEO) coatings, grown on Al-6082 substrates. Conductive paste was used between two back-toback coatings. The values of $K_{\text {true }}$ and $h$ were obtained from the slope and intercept, respectively.

using Eq. (9). The thermal conductivity was found to be $1.63 \pm 0.35 \mathrm{~W} \mathrm{~m}^{-1} \mathrm{~K}^{-1}$. This relatively low value (for alumina) is consistent with certain microstructural features, including a fine grain size, a significant amorphous content [11] and the presence of fine porosity. These measurements incorporate higher error levels than those made with the zirconia coatings. This is a consequence of the high conductivity of the substrate and the low coating thickness, leading respectively to relatively inaccurate heat flux measurements and small temperature drops across the specimen. Nevertheless, the technique looks to be better suited to PEO coatings than any alternative and, indeed, no other method has yet been applied to these coatings.

\section{Conclusions}

The following conclusions can be drawn from this work.

(a) A novel thermal conductivity measurement technique is described. The setup is simple and can be applied to ceramic coatings of different thickness and porosity levels.

(b) The setup is designed to generate steady one-dimensional heat flow through the coating. The heat flux is taken as the average of those through upper and lower substrates, calculated from their thermal conductivities, taking into account any temperature dependence. Any significant difference between these two heat fluxes indicates that the heat flow is not accurately unidirectional, which will introduce errors into the measurements.

(c) The method involves testing samples of different thickness, so that a plot can be constructed of temperature drop over heat flux against specimen thickness. This allows the true thermal conductivity of the sample, $K_{\text {true, }}$, and the interfacial thermal conductance, $h$, to be evaluated.

(d) There is an incentive to maximize the interfacial conductance, since this leads to improved accuracy for the conductivity measurements. This can be done by introducing either compliant pads or (silicone-based) conductive pastes. In both cases, it is helpful for a specified pressure to be applied during measurement, to promote good interfacial contact.

(e) The technique has been validated using fused silica samples of known thermal conductivity $\left(1.46 \mathrm{~W} \mathrm{~m}^{-1} \mathrm{~K}^{-1}\right.$, at $100^{\circ} \mathrm{C}$ ), employing the conductive paste at the interface. The measured values were consistent with this figure, within the expected experimental error.

(f) The thermal conductivity of sprayed yttria-stabilised zirconia (YSZ) thermal barrier coatings (TBCs) was measured to be $1.05 \pm 0.10 \mathrm{~W} \mathrm{~m}^{-1} \mathrm{~K}^{-1}$, using conductive pads and specimens about $0.5-2 \mathrm{~mm}$ in thickness. However, a higher value of $1.38 \pm 0.27 \mathrm{~W} \mathrm{~m}^{-1} \mathrm{~K}^{-1}$ was obtained when the conductive paste was employed. This is attributed to entry of the paste into the extensive surface-connected porosity known to be present in these TBCs. The use of such conductive pastes is not recommended when relatively large surfaceconnected pores are known to be present in the specimen.

(g) The thermal conductivity of plasma electrolytic oxidation (PEO) alumina coatings has also been measured and a value of $1.63 \pm 0.35 \mathrm{~W} \mathrm{~m}^{-1} \mathrm{~K}^{-1}$ was obtained, using the conductive paste. In this case, the experiments were carried out using two back-to-back substrates, with the coatings still attached, and a paste layer between the coatings. There was no significant paste penetration into the specimen with these coatings. Since the coatings are relatively thin $(\$ 100 \mu \mathrm{m})$, the measurement error was greater than with the TBC coatings. Nevertheless, the data are of interest and the errors are probably lower than those expected with all alternative techniques for coatings of this type.

\section{Acknowledgements}

Financial support for this work has come from Sulzer Metco, the Cambridge-MIT Institute (CMI), the Gates Foundation, DSTL and EPSRC, via a Platform Grant.

\section{References}

[1] U.V. Mardolcar, C.A. Nieto de Castro, High Temp. High Press. 24 (1992) 551.

[2] S.R. Mirmira, L.S. Fletcher, J. Thermophys. Heat Transf. 12 (2) (1998) 121.

[3] A.J. Slifka, B.J. Filla, J.M. Phelps, G. Bancke, C.C. Berndt, J. Therm. Spray Technol. 7 (1) (1998) 43.

[4] A.J. Slifka, J. Res. Natl. Inst. Stand. Technol. 105 (4) (2000) 591.

[5] A.J. Slifka, B.J. Filla, J. Res. Natl. Inst. Stand. Technol. 108 (2) (2003) 147.

[6] R. Taylor, J. Phys. E. J. Sci. Instrum. 13 (1980) 1193.

[7] H. Wang, R.B. Dinwiddie, J. Therm. Spray Technol. 9 (2) (2000) 210.

[8] D. Stover, G. Pracht, H. Lehmann, M. Dietrich, J.E. Doring, R. Vassen, J. Therm. Spray Technol. 13 (1) (2004) 76.

[9] R.A. Miller, Surf. Coat. Technol. 30 (1987) 1.

[10] R.A. Miller, J. Therm. Spray Technol. 6 (1) (1997) 35. 
[11] J.A. Curran, T.W. Clyne, Surf. Coat. Technol. 199 (2005) 177.

[12] Heraeus Quarzglas, Quartz Glass for Optics: Data and Properties, Heraeus, Germany, 2004

[13] F.P. Incropera, D.P. Dewitz, Fundamentals of Heat and Mass Transfer, Fifth ed., John Wiley and Sons, 2002.

[14] S.A. Tsipas, I.O. Golosnoy, R. Damani, T.W. Clyne, J. Therm. Spray Technol. 13 (3) (2004) 370.
[15] J.A. Curran, T.W. Clyne, Surf. Coat. Technol. 199 (2005) 168.

[16] Nimonic Alloy 80A Material Datasheet: Physical and Mechanical Properties, Special Metals Corporation, Hereford, UK.

[17] L.A. Abel, R.T. Kiepura, P. Thomas, N.D. Wheaton (Eds.), ASM, Metals Handbook, 10th ed., vol. 1, ASM, Materials Park, Ohio, 1990, p. 197. 